\title{
Lossy Stochastic Game Abstraction with Bounds
}

Tuomas Sandholm, Computer Science Department, Carnegie Mellon University

Satinder Singh, Computer Science \& Engineering, University of Michigan

Abstraction followed by equilibrium finding has emerged as the leading approach to solving games. Lossless abstraction typically yields games that are still too large to solve, so lossy abstraction is needed. Unfortunately, prior lossy game abstraction algorithms have no guarantees on solution quality. We developed a framework that enables the design of lossy game abstraction algorithms with guarantees on solution quality. It simultaneously handles state and action abstraction. We define a measure of reward approximation error and transition probability error achieved by state and action abstraction in stochastic games such that the regret of the equilibrium found in the abstract game when implemented in the original, unabstracted game is upper-bounded by a function of those measures. We then develop the first lossy game abstraction algorithms with bounds on solution quality. Both of them work level-by-level up from the end of the game. One of the algorithms is greedy and the other is an integer linear program. We also prove that the abstraction problem is NP-complete (even with just action abstraction, 2 agents, and a 1-step game), but point out that this does not mean that the game abstraction problems that occur in practice cannot be solved quickly.

Categories and Subject Descriptors: I.2.11 [Distributed Artificial Intelligence]: Multiagent Systems; J.4 [Social and Behavioral Sciences]: Economics; I.2.1 [Artificial intelligence]: Applications and Expert Systems: Games

General Terms: Algorithms, economics, theory

Additional Key Words and Phrases: Equilibrium finding, abstraction, game abstraction, computational game theory, game solving, $\epsilon$-equilibrium

\section{INTRODUCTION}

Game-theoretic solution (equilibrium) concepts provide a rigorous definition of how rational agents should act in multiagent settings. The ability to actually compute such solutions is a key capability in a variety of applications, for example, auctions, exchanges, negotiation, security games, and recreational games such as poker and billiards. Computational techniques for finding such solutions to games have therefore emerged as a central topic in electronic commerce research (e.g., [Littman and Stone 2003; Lipton et al. 2003; Gilpin and Sandholm 2007b; Jiang and Leyton-Brown 2011]) and research in the intersection of economics and computer science at large. This research has focused both on developing new game-solving techniques and on using game-solving techniques to answer important questions in a variety of applications.

The following paradigm has emerged as a leading approach to solving large games. First, the game is abstracted to form a smaller game. Then the abstracted game is solved with some equilibrium-finding algorithm. Finally, that equilibrium is mapped

The authors thank the EC reviewers for many helpful comments. Tuomas Sandholm was supported by the National Science Foundation under grants IIS-0964579, IIS-0905390, and CCF-1101668. Satinder Singh was supported by the National Science Foundation under grants IIS-1148668 and IIS-0905146.

Permission to make digital or hard copies of part or all of this work for personal or classroom use is granted without fee provided that copies are not made or distributed for profit or commercial advantage and that copies show this notice on the first page or initial screen of a display along with the full citation. Copyrights for components of this work owned by others than ACM must be honored. Abstracting with credit is permitted. To copy otherwise, to republish, to post on servers, to redistribute to lists, or to use any component of this work in other works requires prior specific permission and/or a fee. Permissions may be requested from Publications Dept., ACM, Inc., 2 Penn Plaza, Suite 701, New York, NY 10121-0701 USA, fax +1 (212) 869-0481, or permissions@acm.org.

EC'12, June 4-8, 2012, Valencia, Spain. C Copyright 2012 ACM 978-1-4503-1415-2/12/06...\$10.00. 
back into the original, unabstracted game [Gilpin et al. 2008; Schnizlein et al. 2009; Ganzfried et al. 2012].

There are at least three motivations for abstracting games:

(i) The original, unabstracted game can be prohibitively large to be solved with the equilibrium-finding algorithm directly. It may take too much time and/or require too much memory (e.g., [Billings et al. 2003]).

(ii) The original game might be so complex that it is difficult to even model without making the model be an abstraction of reality (e.g., [Wellman 2006]).

(iii) The original game may not fall into a class for which existence of equilibrium is known or may not fall into a class for which an equilibrium-finding algorithm exists; abstraction can remedy this (e.g., [Archibald and Shoham 2009]).

Game abstraction typically takes the form of state abstraction and/or action abstraction. In state abstraction, states of the game are bundled together so the agent(s) whose turn it is to move cannot distinguish among those states. This means that the abstraction pretends that the agent does not know some of the history that the agent actually knows. Therefore, state abstraction is often also referred to as information abstraction. State abstraction means that the agent has to use the same probability mixture over actions in each of the states in the bundled, abstract state. So, state abstraction amounts to a restriction on the agent's strategy space. Action abstraction means that the actions available to an agent at any point in the abstracted game are not necessarily the same as (typically fewer in number than) in the original, unabstracted game.

In early uses of game abstraction, the abstraction was generated manually using domain knowledge (e.g., [Billings et al. 2003]). Similarly, when abstraction is used for reason (ii) or (iii) above, it is typically done manually [Wellman 2006; Archibald and Shoham 2009]. When state abstraction is done for reason (i), it is nowadays usually done automatically using some abstraction algorithm [Gilpin and Sandholm 2007b; Shi and Littman 2002; Gilpin and Sandholm 2006a, 2007a; Gilpin et al. 2007; Zinkevich et al. 2007; Gilpin et al. 2008; Gilpin and Sandholm 2008]. Action abstraction is still typically done by hand [Gilpin et al. 2008; Schnizlein et al. 2009], but that is starting to change [Hawkin et al. 2011].

A key question is how good the abstraction is, that is, how good are the equilibrium strategies from the abstracted game when evaluated in the real, original game. A domain-independent lossless state abstraction algorithm was recently developed for a broad class of games [Gilpin and Sandholm 2007b]. By 'lossless' we mean that the equilibrium from the abstracted game is an exact equilibrium of the original game. For many real games-such as Texas Hold'em poker-the losslessly abstracted game is still too large to solve. Therefore, one needs to employ lossy abstraction. Unfortunately, all prior lossy game abstraction algorithms (e.g., [Shi and Littman 2002; Gilpin and Sandholm 2007b, 2006a, 2007a; Gilpin et al. 2007; Zinkevich et al. 2007; Gilpin et al. 2008; Gilpin and Sandholm 2008; Waugh et al. 2009a,b]) and manually-generated game abstractions (e.g., [Billings et al. 2003]) are lossy without bound. That is, they have no guarantee on how good the equilibrium strategies from the abstracted game will be in the original game. An important open question in this field-and arguably in game solving at large-is whether lossy abstraction algorithms with bounds on solution quality can be devised.

In this paper we develop a framework that enables the design of such algorithms. It simultaneously handles state and action abstraction. It is based on bounding the errors in payoffs as well as errors in transition probabilities, caused by abstraction. Specifically, we define a measure of reward approximation error and transition probability approximation error achieved by state and action abstraction such that the regret of the equilibrium found in the abstract game when implemented in the original, 
unabstracted game is upper-bounded by some function of those measures. The analysis is in some ways similar to that of abstraction in Markov decision processes [Givan et al. 2003; Ravindran 2004; Sorg and Singh 2009], but for the richer-and much more difficult-setting of games.

We then develop the first lossy game abstraction algorithms with bounds on solution quality. We consider finite stochastic general-sum games with any number of agents. We also prove that the abstraction problem is NP-complete even if one looks at one level of the game and does only action abstraction.

The rest of this paper is organized as follows. First, Section 2 illustrates the difficulty of game abstraction: even in a simple game with just action abstraction, a strict refinement of the abstraction can lead to worse solution quality. Then, Section 3 presents our analysis framework. Section 4 proves that state evaluations in the abstract game are near correct in the original game, and Section 5 proves that equilibria from the abstract game have bounded regret in the original game. Section 6 presents abstraction algorithms and analyzes the problem complexity. Finally, Section 7 presents conclusions and discusses future research directions.

\section{EXAMPLE OF NON-MONOTONICITY IN ABSTRACTING GAMES}

Abstraction in games is a much more difficult problem than abstraction in singleagent settings. In short, the difficulty stems from the fact that the opponent(s) may not honor the abstraction when playing. In this section we will illustrate the resulting non-monotonicity in abstracting games.

It is well known that in extensive form games, an equilibrium strategy derived in a finer-grained abstraction can be more exploitable in the original, unabstracted game than an equilibrium strategy derived in a coarser abstraction. Such abstraction pathologies have been demonstrated also experimentally, in relatively small extensive form (artificial poker) games [Waugh et al. 2009a].

The example below shows that abstraction pathologies can occur already in zerosum two-agent one-step stochastic games (i.e., strategic form games). This is the case even if only action abstraction is used.

Example 2.1. Consider a game between an attacker and a defender, with two locations, A and B. There is only one state and the agents move simultaneously, after which the game ends. The attacker has two possible actions, A and B, corresponding to which location he attacks. The defender has three actions, A, B, and BETWEEN, corresponding to where he defends. If the agents choose the same location, the defender wins, in which case he gets payoff 2 and the attacker gets payoff 0 . If the defender choose A or B and the attacker chooses the opposite location, the attacker wins and gets payoff 2 while the defender gets payoff 0 . If the defender choose BETWEEN, the game is a draw: both agents get payoff 1 .

In each equilibrium, the attacker randomizes 50-50 between the locations. The defender plays A with probability $p$ and B with probability $p$, and BETWEEN with probability $1-2 p$. There is an equilibrium for each $p \in[0,0.5]$.

Now, consider an abstraction where the attacker only has action $\mathrm{A}$. The defender would choose $\mathrm{A}$, but that is far from equilibrium in the original, unabstracted game where the attacker would choose B.

Now, consider a strictly coarser abstraction where the attacker only has action A and the defender only has actions B and BETWEEN. The defender would choose BETWEEN in the abstracted game. Interestingly, that choice is an equilibrium strategy in the original, unabstracted game! 


\section{FRAMEWORK}

In this section we will present our analysis framework. We will consider stochastic games that have a finite number of agents and a finite set of possible actions at each state for each agent. We will present everything in the finite-horizon undiscounted setting (we discuss the generalization to the infinite-horizon discounted setting later).

If the stochastic game is given as a directed graph with cycles, then we conceptually attach time to the states so that if a state can be reached via two different-length paths in the graph, we consider that state to be two different states. However, if a state can be reached via different paths of the same length, we do not duplicate the state. (This guarantees that the number of states in the game is linear in the number of steps that the game is played.) As a result, our game representation is a layered directed acyclic graph (LDAG). Layer 1 consists of the start state, layer 2 consists of the states reachable in one step, and so on. Throughout the paper we will refer to the height of a state, which is equivalent to layer, except counting from the other direction, so leaves are at height 1 , states just before the leaves are at height 2 , and so on. We will use the word level as a synonym for height.

Consider two stochastic games: the original, unabstracted one, $M$, and the abstracted one, $M^{\prime}$. Each of the two games has $n$ agents.

We will denote the elements of $M$ as follows. Its state space is $S$. The set of states at height $j$ is $S_{j} \subset S$ (note, for all $j \neq k, S_{j} \cap S_{k}=\phi$ ). In state $s \in S$ the action space for Agent $i$ is $A_{i}(s)$ and the joint action space of all $n$ agents is $A(s)$. In state $x \in S_{j}$, on taking joint action a $\in A(x)$, the payoff to Agent $i$ is $R_{i}(x, \mathbf{a})$ and the next state is $y \in S_{j-1}$ with probability $T(x, \mathbf{a}, y)$. Without loss of generality, we assume that all the payoffs are nonnegative (one can always add a constant to all payoffs while keeping the game strategically equivalent). The action of Agent $i$ in joint action a is denoted $\mathbf{a}_{i}$.

The elements of $M^{\prime}$ are denoted exactly as for $M$ above except that a "prime" superscript is used for all the elements, e.g., the state space of $M^{\prime}$ is denoted $S^{\prime}$.

\subsection{State and action abstraction functions}

We will define a state abstraction function $h: S \rightarrow S^{\prime}$. Also, for all Agents $i$ and for all $s \in S$, we will define an action abstraction function $g_{s, i}: A_{i}(s) \rightarrow A_{i}^{\prime}(h(s))$. We will have these functions be surjections, so $\left|S^{\prime}\right| \leq|S|$ and for all agents $i$ and for all $s \in S$, $\left|A_{i}^{\prime}(h(s))\right| \leq\left|A_{i}(s)\right|$. The intent is that $M^{\prime}$ is smaller than $M$ and thus we will refer to $M^{\prime}$ as the abstract game. When we wish to emphasize this aspect we will attach the prefix "abstract" to the elements associated with $M^{\prime}$.

State $s$ in $M$ maps to abstract state $h(s)$ in $M^{\prime}$. The set of states that map to abstract state $s^{\prime}$ is denoted $h^{-1}\left(s^{\prime}\right) \subset S$ (i.e., $h$ induces a partition of $S$ ).

Action a in state $s$ in $M$ maps to abstract action $g_{s}(\mathbf{a})$ in abstract state $h(s)$ in $M^{\prime}$. In state $s$, the set of actions for Agent $i$ that map to abstract action $\mathbf{a}_{i}^{\prime}$ is denoted $g_{s, i}^{-1}\left(\mathbf{a}_{i}^{\prime}\right)$, and the set of joint actions that map to abstract joint action $\mathbf{a}^{\prime}$ is denoted $g_{s}^{-1}\left(\mathbf{a}^{\prime}\right)$. Note that the action abstraction functions are factored by both state and action, i.e., $g_{s}^{-1}\left(\mathbf{a}^{\prime}\right)=\times_{i=1}^{n} g_{s, i}^{-1}\left(\mathbf{a}_{i}^{\prime}\right)$ (where the symbol $\times$ denotes cross-product).

An abstract strategy $\sigma^{\prime}$ maps abstract states of $M^{\prime}$ to abstract joint-actions in $M^{\prime}$. An abstract strategy for Agent $i$ in $M^{\prime}$ is denoted $\sigma_{i}^{\prime}$.

How does one take a strategy $\sigma^{\prime}$ in the abstract game $M^{\prime}$ and apply it in $M$ ? There are many ways of doing this "lifting". That is, for state $s$ the probability $\sigma_{i}^{\prime}\left(\mathbf{a}_{i}^{\prime} \mid h(s)\right)$ of abstract action $\mathbf{a}_{i}^{\prime}$ by Agent $i$ can be apportioned arbitrarily among the actions $g_{s, i}^{-1}\left(\mathbf{a}_{i}^{\prime}\right)$ in the lifted strategy.

Definition 3.1. (Strategy Lifting) Given an abstract strategy $\sigma_{i}^{\prime}$ for Agent $i$ in $M^{\prime}$, a lifted strategy for Agent $i$ in $M$, denoted $\sigma_{i}^{\uparrow \sigma_{i}^{\prime}}$ is any strategy in $M$ that satisfies the 
following conditions: for all $s \in S$, for all $i$, for all $\mathbf{a}_{i}^{\prime} \in A_{i}^{\prime}$,

$$
\sum_{\mathbf{a}_{i} \in g_{s, i}^{-1}\left(\mathbf{a}_{i}^{\prime}\right)} \sigma_{i}^{\uparrow \sigma_{i}^{\prime}}\left(\mathbf{a}_{i} \mid s\right)=\sigma_{i}^{\prime}\left(\mathbf{a}_{i}^{\prime} \mid h(s)\right) \text {. }
$$

Furthermore, $\sigma_{i}^{\uparrow \sigma^{\prime}}$ is the same as $\sigma_{i}^{\uparrow \sigma_{i}^{\prime}}$, and $\sigma^{\uparrow \sigma^{\prime}}$ assigns $\sigma_{i}^{\uparrow \sigma_{i}^{\prime}}$ to each $i$.

Conversely, how does one take a strategy $\sigma$ defined in $M$ and apply it in $M^{\prime}$ ? One cannot do this in general. However, by construction, the strategy $\sigma^{\uparrow \sigma^{\prime}}$ defined in $M$ can always be applied in $M^{\prime}$.

\subsection{Value functions for the original and abstract game}

We define value functions for the games as follows. For joint strategy $\sigma$ in $M$, for Agent $i$ the value of a non-terminal state $x \in S_{k>1}$ is

$$
V_{i}^{\sigma}(x)=\sum_{\mathbf{a} \in A(x)} \sigma(\mathbf{a} \mid x)\left[R_{i}(x, \mathbf{a})+\sum_{y \in S_{k-1}} T(x, \mathbf{a}, y) V_{i}^{\sigma}(y)\right]
$$

where for terminal states $x \in S_{1}, V_{i}^{\sigma}(x)=\sum_{\mathbf{a} \in A(x)} \sigma(\mathbf{a} \mid x) R_{i}(x, \mathbf{a})$. For joint abstract strategy $\sigma^{\prime}$ in $M^{\prime}$, the value of Agent $i$ in abstract non-terminal state $x^{\prime} \in S_{k>1}^{\prime}$ is

$$
W_{i}^{\sigma^{\prime}}\left(x^{\prime}\right)=\sum_{\mathbf{a}^{\prime} \in A^{\prime}\left(x^{\prime}\right)} \sigma^{\prime}\left(\mathbf{a}^{\prime} \mid x^{\prime}\right)\left[R_{i}^{\prime}\left(x^{\prime}, \mathbf{a}^{\prime}\right)+\sum_{y^{\prime} \in S_{k-1}^{\prime}} T^{\prime}\left(x^{\prime}, \mathbf{a}^{\prime}, y^{\prime}\right) W_{i}^{\sigma^{\prime}}\left(y^{\prime}\right)\right]
$$

where for terminal states $x^{\prime} \in S_{1}^{\prime}, W_{i}^{\sigma^{\prime}}\left(x^{\prime}\right)=\sum_{\mathbf{a}^{\prime} \in A^{\prime}\left(x^{\prime}\right)} \sigma^{\prime}\left(\mathbf{a}^{\prime} \mid x^{\prime}\right) R_{i}^{\prime}\left(x^{\prime}, \mathbf{a}^{\prime}\right)$. Note that we will use $V^{\prime}$ 's to denote values in $M$ and $W^{\prime}$ 's to denote values in $M^{\prime}$. Finally, define the largest, over all states in LDAG-level $k$, value obtained in $M^{\prime}$ under abstract strategy $\sigma^{\prime}$ as

$$
\bar{W}_{k, i}^{\sigma^{\prime}} \stackrel{\text { def }}{=} \max _{x^{\prime} \in S_{k}^{\prime}} W_{i}^{\sigma^{\prime}}\left(x^{\prime}\right) \quad \text { and define } \quad \bar{W}_{k}^{\sigma^{\prime}} \stackrel{\text { def }}{=} \max _{i} \bar{W}_{k, i}^{\sigma^{\prime}} \quad \text { and } \quad \bar{W}^{\sigma^{\prime}} \stackrel{\text { def }}{=} \max _{k} \bar{W}_{k}^{\sigma^{\prime}} .
$$

\subsection{Reward-approximation and transition-approximation error bounds}

Next we define reward-approximation error bounds in the mapping from $M$ to $M^{\prime}$, as a function of LDAG-level and agent:

$$
\epsilon_{k, i}^{R} \stackrel{\text { def }}{=} \max _{s \in S_{k}, \mathbf{a} \in A(s)}\left|R_{i}(s, \mathbf{a})-R_{i}^{\prime}\left(h(s), g_{s}(\mathbf{a})\right)\right|,
$$

then as a function of just LDAG-level:

$$
\epsilon_{k}^{R} \stackrel{\text { def }}{=} \max _{i}\left[\epsilon_{k, i}^{R}\right]
$$

and finally as a global bound on the entire game:

$$
\epsilon^{R}=\max _{k} \epsilon_{k}^{R} .
$$

Similarly we can define transition-approximation error bounds, first as a function of LDAG-level and next abstract state:

$$
\epsilon_{k}^{T}\left(x^{\prime} \in S_{k-1}^{\prime}\right) \stackrel{\text { def }}{=} \max _{s \in S_{k}, \mathbf{a} \in A(s)}\left|\sum_{x \in h^{-1}\left(x^{\prime}\right)} T(s, \mathbf{a}, x)-T^{\prime}\left(h(s), g_{s}(\mathbf{a}), x^{\prime}\right)\right|,
$$

then as a function of just LDAG-level:

$$
\epsilon_{k}^{T} \stackrel{\text { def }}{=} \sum_{x^{\prime} \in S_{k-1}^{\prime}} \epsilon_{k}^{T}\left(x^{\prime}\right)
$$


and finally as a global bound on the entire game:

$$
\epsilon^{T} \stackrel{\text { def }}{=} \max _{k} \epsilon_{k}^{T} \text {. }
$$

\section{EVALUATIONS IN THE ABSTRACT GAME ARE NEAR CORRECT IN THE ORIGINAL GAME}

Our first result is to show that the evaluation of an abstract strategy in $M^{\prime}$ is not too far from the evaluation of any corresponding lifted strategy in $M$. The key insight here is that we can state such an approximation result for entire, arbitrary strategy profiles (which contain one strategy for each agent)—rather than, say, studying equilibrium properties or best-response properties of individual strategies or strategy profiles. We will leverage this insight (as embodied in Proposition 4.1) when we present our gametheoretic results in the rest of the paper.

Proposition 4.1. $\forall \sigma^{\prime}, \forall s \in S_{k}, \forall i$,

$$
\begin{aligned}
& \text { 4.1. } \forall \sigma^{\prime}, \forall s \in S_{k}, \forall i, \\
& \left|V_{i}^{\sigma^{\uparrow \sigma^{\prime}}}(s)-W_{i}^{\sigma^{\prime}}(h(s))\right| \leq f_{k, i} \stackrel{\text { def }}{=} \sum_{j=1}^{k} \epsilon_{j, i}^{R}+\sum_{j=1}^{k-1} \bar{W}_{j, i}^{\sigma^{\prime}} \epsilon_{j+1}^{T} .
\end{aligned}
$$

PROOF. By induction on the height of the LDAG.

Base case (terminal nodes of the LDAG): $\forall x \in S_{1}$,

$$
\begin{aligned}
& V_{i}^{\sigma^{\uparrow \sigma^{\prime}}}(x) \stackrel{\text { def }}{=} \sum_{\mathbf{a} \in A(x)} \sigma^{\uparrow \sigma^{\prime}}(\mathbf{a} \mid x) R_{i}(x, \mathbf{a}) \\
& \leq \sum_{\mathbf{a} \in A(x)} \sigma^{\uparrow \sigma^{\prime}}(\mathbf{a} \mid x)\left[R_{i}^{\prime}\left(h(x), g_{x}(\mathbf{a})\right)+\epsilon_{1, i}^{R}\right] ; \text { by Equation } 3 \\
& =\epsilon_{1, i}^{R}+\sum_{\mathbf{a}^{\prime} \in A^{\prime}(h(x))} \sum_{\mathbf{a} \in g_{x}^{-1}\left(\mathbf{a}^{\prime}\right)} \sigma^{\uparrow \sigma^{\prime}}(\mathbf{a} \mid x) R_{i}^{\prime}\left(h(x), g_{x}(\mathbf{a})\right) \\
& =\epsilon_{1, i}^{R}+\sum_{\mathbf{a}^{\prime} \in A^{\prime}(x)} \sigma^{\prime}\left(\mathbf{a}^{\prime} \mid h(x)\right) R_{i}^{\prime}\left(h(x), \mathbf{a}^{\prime}\right) ; \text { by Definition } 3.1 \\
& =\epsilon_{1, i}^{R}+W_{i}^{\sigma^{\prime}}(h(x)) \\
& W_{i}^{\sigma^{\prime}}(h(x)) \stackrel{\text { def }}{=} \sum_{\mathbf{a}^{\prime} \in A^{\prime}(h(x))} \sigma^{\prime}\left(\mathbf{a}^{\prime} \mid h(x)\right) R_{i}^{\prime}\left(h(x), \mathbf{a}^{\prime}\right) \\
& =\sum_{\mathbf{a}^{\prime} \in A^{\prime}(h(x))}\left(\sum_{\mathbf{a} \in g_{x}^{-1}\left(\mathbf{a}^{\prime}\right)} \sigma^{\uparrow \sigma^{\prime}}(\mathbf{a} \mid x)\right) R_{i}^{\prime}\left(h(x), \mathbf{a}^{\prime}\right) ; \text { by Definition } 3.1 \\
& \leq \sum_{\mathbf{a}^{\prime} \in A^{\prime}(h(x))} \sum_{\mathbf{a} \in g_{s}^{-1}\left(\mathbf{a}^{\prime}\right)} \sigma^{\uparrow \sigma^{\prime}}(\mathbf{a} \mid x)\left[R_{i}(x, \mathbf{a})+\epsilon_{1, i}^{R}\right] ; \text { by Equation } 3 \\
& =V_{i}^{\sigma^{\uparrow \sigma^{\prime}}}(x)+\epsilon_{1, i}^{R}
\end{aligned}
$$

Putting the two pieces (Equations 9 and 10) together, we have $W_{i}^{\sigma^{\prime}}(h(x))-\epsilon_{1, i}^{R} \leq$ $V_{i}^{\sigma^{\uparrow \sigma^{\prime}}}(x) \leq W_{i}^{\sigma^{\prime}}(h(x))+\epsilon_{1, i}^{R}$

Step 2: We assume that the proposition holds for $x \in S_{\leq k}$.

Step 3: We prove that the assumption from Step 2 implies that the proposition holds for $x \in S_{k+1}$. We will only show one side of the two-sided inequality in the Proposition. The other side is similarly derived.

$$
V_{i}^{\sigma^{\uparrow \sigma^{\prime}}}(x) \stackrel{\text { def }}{=} \sum_{\mathbf{a} \in A(x)} \sigma^{\uparrow \sigma^{\prime}}(\mathbf{a} \mid x)\left[R_{i}(x, \mathbf{a})+\sum_{y \in S_{k}} T(x, \mathbf{a}, y) V_{i}^{\sigma^{\uparrow \sigma^{\prime}}}(y)\right]=\text { term } 1+\text { term } 2
$$




$$
\begin{aligned}
\text { term } 1 & \stackrel{\text { def }}{=} \sum_{\mathbf{a} \in A(x)} \sigma^{\uparrow \sigma^{\prime}}(\mathbf{a} \mid x) R_{i}(x, \mathbf{a}) \leq \sum_{\mathbf{a} \in A(x)} \sigma^{\uparrow \sigma^{\prime}}(\mathbf{a} \mid x)\left[R_{i}^{\prime}\left(h(x), \mathbf{a}^{\prime}\right)+\epsilon_{k+1, i}^{R}\right] \\
& =\epsilon_{k+1, i}^{R}+\sum_{\mathbf{a}^{\prime} \in A^{\prime}(h(x))} \sum_{\mathbf{a} \in g_{x}^{-1}\left(\mathbf{a}^{\prime}\right)} \sigma^{\uparrow \sigma^{\prime}}(\mathbf{a} \mid x) R_{i}^{\prime}\left(h(x), \mathbf{a}^{\prime}\right) \\
& =\epsilon_{k+1, i}^{R}+\sum_{\mathbf{a}^{\prime} \in A^{\prime}(h(x))} \sigma^{\prime}\left(\mathbf{a}^{\prime} \mid h(x)\right) R_{i}^{\prime}\left(h(x), \mathbf{a}^{\prime}\right)
\end{aligned}
$$

$$
\begin{aligned}
\text { term2 } & \stackrel{\text { def }}{=} \sum_{\mathbf{a} \in A(x)} \sigma^{\uparrow \sigma^{\prime}}(\mathbf{a} \mid x) \sum_{y \in S_{k}} T(x, \mathbf{a}, y) V_{i}^{\sigma^{\uparrow \sigma^{\prime}}}(y) \\
& \leq \sum_{\mathbf{a} \in A(x)} \sigma^{\uparrow \sigma^{\prime}}(\mathbf{a} \mid x) \sum_{y \in S_{k}} T(x, \mathbf{a}, y)\left[W_{i}^{\sigma^{\prime}}(h(y))+f_{k, i}\right] ; \text { by induction Step 2 } \\
& =f_{k, i}+\sum_{\mathbf{a} \in A(x)} \sigma^{\uparrow \sigma^{\prime}}(\mathbf{a} \mid x) \sum_{y \in S_{k}} T(x, \mathbf{a}, y) W_{i}^{\sigma^{\prime}}(h(y)) \\
& =f_{k, i}+\sum_{\mathbf{a} \in A(x)} \sigma^{\uparrow \sigma^{\prime}}(\mathbf{a} \mid x) \sum_{y^{\prime} \in S_{k}^{\prime}} \sum_{y \in h^{-1}\left(y^{\prime}\right)} T(x, \mathbf{a}, y) W_{i}^{\sigma^{\prime}}\left(y^{\prime}\right) \\
& \leq f_{k, i}+\sum_{\mathbf{a} \in A(x)} \sigma^{\uparrow \sigma^{\prime}}(\mathbf{a} \mid x) \sum_{y^{\prime} \in S_{k}^{\prime}}\left(T^{\prime}\left(h(x), g_{x}(\mathbf{a}), y^{\prime}\right)+\epsilon_{k+1}^{T}\left(y^{\prime}\right)\right) W_{i}^{\sigma^{\prime}}\left(y^{\prime}\right) \\
& =f_{k, i}+\sum_{y^{\prime} \in S_{k}^{\prime}} W_{i}^{\sigma^{\prime}}\left(y^{\prime}\right) \epsilon_{k+1}^{T}\left(y^{\prime}\right)+\sum_{\mathbf{a} \in A(x)} \sigma^{\uparrow \sigma^{\prime}}(\mathbf{a} \mid x) \sum_{y^{\prime} \in S_{k}^{\prime}} T^{\prime}\left(h(x), g_{x}(\mathbf{a}), y^{\prime}\right) W_{i}^{\sigma^{\prime}}\left(y^{\prime}\right) \\
& \leq f_{k, i}+\bar{W}_{k, i}^{\sigma^{\prime}} \epsilon_{k+1}^{T}+\sum_{\mathbf{a} \in A(x)} \sigma^{\uparrow \sigma^{\prime}}(\mathbf{a} \mid x) \sum_{y^{\prime} \in S_{k}^{\prime}} T^{\prime}\left(h(x), g_{x}(\mathbf{a}), y^{\prime}\right) W_{i}^{\sigma^{\prime}}\left(y^{\prime}\right) \\
& =f_{k, i}+\bar{W}_{k, i}^{\sigma^{\prime}} \epsilon_{k+1}^{T}+\sum_{\mathbf{a}^{\prime} \in A^{\prime}(h(x))} \sum_{y^{\prime} \in S_{k}^{\prime} \mathbf{a} \in g_{x}^{-1}\left(\mathbf{a}^{\prime}\right)} \sum_{\sigma^{\uparrow \sigma^{\prime}}(\mathbf{a} \mid x) T^{\prime}\left(h(x), \mathbf{a}^{\prime}, y^{\prime}\right) W_{i}^{\sigma^{\prime}}\left(y^{\prime}\right)} \underbrace{}_{\mathbf{a}^{\prime} \in A^{\prime}(h(x))} \mathbf{a}^{\prime} \mid h(x)) \sum_{y^{\prime} \in S_{k}^{\prime}} T^{\prime}\left(h(x), \mathbf{a}^{\prime}, y^{\prime}\right) W_{i}^{\sigma^{\prime}}\left(y^{\prime}\right)
\end{aligned}
$$

Using Equations 12 and 13 we get the following:

$$
\begin{aligned}
V_{i}^{\sigma^{\uparrow \sigma^{\prime}}}(x) \leq & f_{k, i}+\epsilon_{k+1, i}^{R}+\bar{W}_{k, i}^{\sigma^{\prime}} \epsilon_{k+1}^{T} \\
& +\sum_{\mathbf{a}^{\prime} \in A^{\prime}(h(x))} \sigma^{\prime}\left(\mathbf{a}^{\prime} \mid h(x)\right)\left[R_{i}^{\prime}\left(h(x), \mathbf{a}^{\prime}\right)+\sum_{y^{\prime} \in S_{k}^{\prime}} T^{\prime}\left(h(x), \mathbf{a}^{\prime}, y^{\prime}\right) W_{i}^{\sigma^{\prime}}\left(y^{\prime}\right)\right] \\
= & W_{i}^{\sigma^{\prime}}(h(x))+\left(\epsilon_{k+1, i}^{R}+\bar{W}_{k, i}^{\sigma^{\prime}} \epsilon_{k+1}^{T}\right)+f_{k, i} .
\end{aligned}
$$

This sets up the recursion

$$
f_{k+1, i}=\epsilon_{k+1, i}^{R}+\bar{W}_{k, i}^{\sigma^{\prime}} \epsilon_{k+1}^{T}+f_{k, i}
$$

where $f_{0, i}=0$, and $\epsilon_{1}^{T}=0$, and $\bar{W}_{0, i}^{\sigma^{\prime}}=0$.

Proposition 4.1 was presented in a manner that allowed the use of level and agent dependent reward and transition error bounds. By using the largest error bounds across agents within a level, we present a simpler form of the result in the following corollary. 
Corollary 4.2. $\forall \sigma^{\prime}, \forall s \in S_{k}, \forall i, \quad\left|V_{i}^{\sigma^{\uparrow \sigma^{\prime}}}(s)-W_{i}^{\sigma^{\prime}}(h(s))\right| \leq k\left[\epsilon^{R}+\bar{W}_{k}^{\sigma^{\prime}} \epsilon^{T}\right]$.

Observe that the overall error in value functions is quadratic in the height of the LDAG (the additional dependence on $k$ comes from the linear dependence on $k$ of $\bar{W}_{k}^{\sigma^{\prime}}$ ).

\section{EQUILIBRIA FROM ABSTRACT GAME HAVE BOUNDED REGRET IN ORIGINAL GAME}

Here we apply game-theoretic reasoning while leveraging the approximation results from the previous section that apply to all strategy profiles. We will show that any subgame perfect Nash equilibrium (SPNE) in an abstract game $M^{\prime}$ is an approximate SPNE in the original game $M$. In particular, we will show how the regret of any SPNE of $M^{\prime}$ when lifted to $M$ is bounded in terms of the reward and transition abstraction error bounds of the state and action abstraction functions that map $M$ to $M^{\prime}$.

THEOREM 5.1. For any subgame perfect Nash equilibrium (SPNE) strategy $\sigma^{*}$ in $M^{\prime}$, any corresponding lifted joint strategy $\sigma^{\uparrow \sigma^{\prime *}}$ in $M$ has the property that

$$
\forall i, \forall s \in S_{k}, \forall \pi_{i} \in S \rightarrow A_{i}(S) \quad: \quad V_{i}^{\left\langle\pi_{i}, \sigma_{-i}^{\left.\uparrow \sigma^{\prime *}\right\rangle}\right.}(s) \leq V_{i}^{\sigma^{\uparrow \sigma^{\prime *}}}(s)+2 k f_{k, i}
$$

where $\left\langle\pi_{i}, \sigma_{-i}^{\uparrow \sigma^{*}}\right\rangle$ is the joint strategy in $M$ that results from Agent $i$ unilaterally deviating from $\sigma^{\uparrow \sigma^{\prime *}}$ to pure strategy $\pi_{i}$, and $f_{k, i}$ is as defined in Proposition 4.1.

Proof. By induction on the height of the LDAG (as in the proof for Proposition 4.1). Note that unlike strategy $\sigma^{\uparrow \sigma^{\prime *}}$, the strategy $\left\langle\pi_{i}, \sigma_{-i}^{\uparrow \sigma^{\prime *}}\right\rangle$ is not implementable in the abstract game $M^{\prime}$. We will also need the following definition of an adaptation of $\sigma^{\uparrow \sigma^{\prime}}$. that is consistent with the action of $\pi_{i}$ on state $s$ and hence is constrained to take the same action in all states in $h(s)$ to be implementable in $M^{\prime}$, i.e., the only change from $\sigma^{\uparrow \sigma^{\prime *}}$ is that

$$
\sigma_{i}^{\uparrow \sigma_{\pi_{i}}^{\prime *}(s) \mid s}\left(\pi_{i}(s) \mid x \in h(s)\right)=1
$$

where the corresponding abstract strategy is denoted $\sigma_{\pi_{i}}^{*}(s) \mid s$.

Base case (terminal nodes of the LDAG): We prove the theorem holds for $s \in S_{1}$.

Suppose pure strategy $\pi_{i}$ is such that for some $s \in S_{1}, V_{i}^{\left\langle\pi_{i}, \sigma_{-i}^{\left.\uparrow \sigma^{\prime *}\right\rangle}\right.}(s)>V_{i}^{\sigma^{\uparrow \sigma^{\prime *}}}(s)+2 f_{1, i}$. Define $\sigma_{M, \pi_{i}(s) \mid s}^{\prime *}$ to be the minimal adaptation of $\sigma_{M}^{\prime *}$ as in the theorem statement. Now,

$$
V_{i}^{\left\langle\pi_{i}, \sigma_{-i}^{\left.\uparrow \sigma^{\prime *}\right\rangle}\right.}(s)=\sum_{\mathbf{a}_{-i} \in A_{-i}} \sigma_{-i}^{\uparrow \sigma^{\prime *}}\left(\mathbf{a}_{-i} \mid s\right) R_{i}\left(s,\left\langle\pi_{i}(s), \mathbf{a}_{-i}\right\rangle\right)=V_{i}^{\sigma^{\uparrow \sigma_{\pi_{i}}^{\prime *}(s) \mid s}}(s) .
$$

We get a contradiction by the following chain:

$$
\begin{aligned}
W_{i}^{\sigma_{\pi_{i}(s) \mid s}^{\prime *}}(h(s)) & \geq V_{i}^{\sigma^{\uparrow \sigma_{\pi_{i}}^{\prime *}(s) \mid s}}(s)-f_{1, i} ; \text { by Proposition } 4.1 \\
& =V_{i}^{\left\langle\pi_{i}, \sigma_{-i}^{\left.\uparrow \sigma^{\prime *}\right\rangle}\right.}(s)-f_{1, i} ; \text { by Equation } 16 \\
& >\left(V_{i}^{\sigma^{\uparrow \sigma^{\prime *}}}(s)+2 f_{1, i}\right)-f_{1, i} ; \text { by supposition } \\
& \geq\left(\left({W_{i}^{\sigma^{\prime *}}}^{\sigma^{\prime}}(h(s))-f_{1, i}\right)+2 f_{1, i}\right)-f_{1, i} ; \text { by Proposition } 4.1 \\
& =W_{i}^{\sigma^{\prime *}}(h(s))
\end{aligned}
$$


which is a contradiction because $\sigma^{*}$ is an SPNE in $M^{\prime}$.

Step 2: We assume the theorem holds for $s \in S_{\leq k}$.

Step 3: We prove that the assumption from Step 2 implies that the theorem holds for $s \in S_{k+1}$.

Suppose $\pi_{i}$ is such that for some $s \in S_{k+1}, V_{i}^{\left\langle\pi_{i}, \sigma_{-i}^{\left.\uparrow \sigma^{\prime *}\right\rangle}\right.}(s)>V_{i}^{\sigma^{\uparrow \sigma^{\prime *}}}(s)+2(k+1) f_{k+1, i}$. Now,

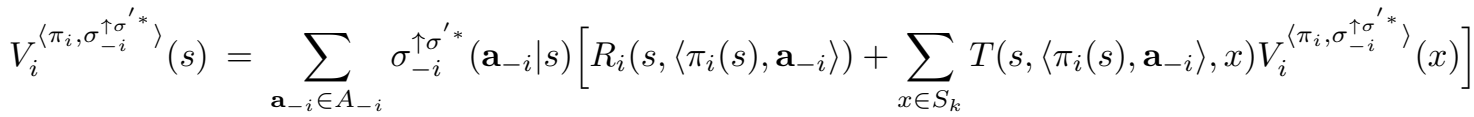

$$
\begin{aligned}
& =\sum_{\mathbf{a}_{-i} \in A_{-i}} \sigma_{-i}^{\uparrow \sigma^{\prime *}}\left(\mathbf{a}_{-i} \mid s\right) R_{i}\left(s,\left\langle\sigma_{i}^{\uparrow \sigma_{\pi_{i}(s) \mid s}^{\prime *}}(s), \mathbf{a}_{-i}\right\rangle\right)
\end{aligned}
$$

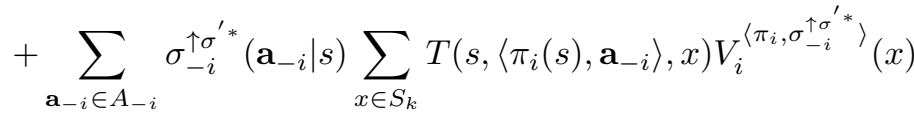

$$
\begin{aligned}
& \leq \sum_{\mathbf{a}_{-i} \in A_{-i}} \sigma_{-i}^{\uparrow \sigma^{\prime *}}\left(\mathbf{a}_{-i} \mid s\right) R_{i}\left(s,\left\langle\sigma_{i}^{\uparrow \sigma_{\pi_{i}(s) \mid s}^{\prime *}}(s), \mathbf{a}_{-i}\right\rangle\right) \\
& +\sum_{\mathbf{a}_{-i} \in A_{-i}} \sigma_{-i}^{\uparrow \sigma^{\prime *}}\left(\mathbf{a}_{-i} \mid s\right) \sum_{x \in S_{k}} T\left(s,\left\langle\pi_{i}(s), \mathbf{a}_{-i}\right\rangle, x\right)\left[V_{i}^{\sigma^{\uparrow \sigma_{\pi_{i}(s) \mid s}^{\prime *}}}(x)+2 k f_{k, i}\right] \\
& =V_{i}^{\sigma^{\uparrow \sigma^{\prime *} *(s) \mid s}}(s)+2 k f_{k, i} \\
& \leq W_{i}^{\sigma_{\pi_{i}(s) \mid s}^{\prime *}}(h(s))+f_{k+1, i}+2 k f_{k, i} \text {. }
\end{aligned}
$$

We get a contradiction by the chain

$$
\begin{aligned}
W_{i}^{\sigma_{\pi_{i}(s) \mid s}^{\sigma^{*}}}(h(s)) & \geq V_{i}^{\left\langle\pi_{i}, \sigma_{-i}^{\left.\uparrow \sigma^{\prime *}\right\rangle}\right.}(s)-f_{k+1, i}-2 k f_{k, i} ; \text { by Equation } 17 \\
& >\left[V_{i}^{\sigma^{\uparrow \sigma^{\prime *}}}(s)+2(k+1) f_{k+1, i}\right]-f_{k+1, i}-2 k f_{k, i} ; \text { by supposition } \\
& =V_{i}^{\sigma^{\uparrow \sigma^{\prime *}}}(s)+2 k\left(f_{k+1, i}-f_{k, i}\right)+f_{k+1, i} \\
& \geq\left[W_{i}^{\sigma^{\prime *}}(h(s))-f_{k+1, i}\right]+2 k\left(f_{k+1, i}-f_{k, i}\right)+f_{k+1, i} ; \text { by Proposition } 4.1 \\
& =W_{i}^{\sigma^{\prime *}}(h(s))+2 k\left(f_{k+1, i}-f_{k, i}\right)
\end{aligned}
$$

which is a contradiction because by construction in Proposition 4.1, $f_{k+1, i} \geq f_{k, i}$.

The regret at height $k$ in the regret bound of Theorem 5.1 depends cubically on $k$ (there is a dependence on $k$ in Equation 15 and a further multiplicative dependence on $k^{2}$ in the $f_{k, i}$ term as defined in the statement of Proposition 4.1).

\subsection{Extension to infinite-horizon discounted stochastic games}

In the single-agent Markov Decision Process (MDP) counterpart to stochastic games there is a long history (e.g., [Kearns et al. 1999]) of using finite-horizon analyses as the basis for an analysis of the discounted infinite-horizon MDP. Here we very briefly sketch the same argument-structure applied to our stochastic game setting. Suppose we pick a finite horizon of $L$ and find abstractions as specified in our paper so that the regret at the root node (where the regret is the largest) is $\epsilon$. Now consider any 
extension of the finite horizon strategy profile to an infinite-horizon strategy profile, e.g., by always selecting the same arbitrary strategy profile at every time step after time $L$. What is the maximum additional regret at the root node? The additional regret is upper bounded by the fraction $\gamma^{L} \frac{R_{\max }}{1-\gamma}$, where $R_{\max }$ is the difference between the largest reward and smallest reward. Crucially, this additional regret term decreases exponentially with $L$ and so by making $L$ large enough we can make this additional regret as small as we want (in particular, we can apportion a fraction of the overall error guarantee we seek to the error due to the finite-horizon approximation). We leave the detailed derivation of this result to future work.

\section{ABSTRACTION ALGORITHMS}

Here we develop algorithms for automatically constructing an abstract game $M^{\prime}$ from the original game $M$ with the guarantee that an SPNE in $M^{\prime}$ when lifted and implemented in $M$ will be an approximate SPNE in $M$ (cf. Theorem 5.1).

\subsection{Bottom-up single-pass level-by-level greedy abstraction algorithm}

We start by describing a greedy, bottom-up, single-pass algorithm. Given an overall approximation bound on the quality of the SPNE to be found by constructing $M^{\prime}$, there is a challenging and interesting question of how to apportion the approximation bound into the $\epsilon$ 's needed for the different levels of the game (see Equations 3,4,6,7) and indeed more finely to the different state and action abstractions. For now, we assume that we have done this apportionment by working backwards from our theory in Section 4. One simple way to do this would be to assume that the $\epsilon^{R}$ 's and $\epsilon^{T}$ 's are the same for all levels and then solving for these two constants from Equation 15. Regardless of how we obtain them, we will present an algorithm which will take as input $\epsilon_{k}^{R}$ and $\epsilon_{k}^{T}$ for every level $k$ separately.

Our algorithm for constructing $M^{\prime}$ proceeds bottom-up, layer by layer as shown in Algorithm 3. For each layer, first the action-abstractions ( $g$ 's) are constructed greedily and then the state-abstractions ( $h$ 's) are constructed greedily. This ordering of actions first and states second was a choice and one could get a similar algorithm by doing this in the reverse order. The size of $M^{\prime}$ would in general depend on this choice and which choice is better would depend on the details of $M$.

Action Abstractions. Algorithm 1 shows the procedure for obtaining action abstractions for any specific state given the state abstractions at the level below. It takes a state $s$ as input, cycles through each agent (line 1), for each agent considers every action in $A(s)$ (line 2), and then buckets the actions with a prototype-action for each bucket. Buckets get assigned by checking both that the rewards are similar (lines 810) and that transitions are similar (lines 12-14), where similarity is defined by the $\epsilon$ and $\epsilon^{\prime}$ input parameters respectively. The function $g_{s, i}\left(a_{i} \in A_{i}(s)\right)$ maps action $a_{i}$ to the prototype-action for its bucket. Algorithm 1 is called separately for every state (see lines 3-5 of Algorithm 3).

State Abstractions. Algorithm 2 shows the procedure for obtaining state abstractions for any level given the action abstractions already obtained at that level as well as the state abstractions at the level below. It cycles through every state $s$ at that level (line 1) and compares it to every state $x$ it has already mapped to a bucket before (line 4). The comparisons involve finding a one-to-one correspondence (line 9) if one exists between the abstract actions of $s$ and the abstract actions in the prototype state corresponding to $x$ that satisfies reward similarity (line 10) and satisfies transition similarity (lines 11-12), where similarity is defined by the $\epsilon$ and $\epsilon^{\prime}$ input parameters respectively. Such a one-to-one correspondence can be found via a bipartite-matching 


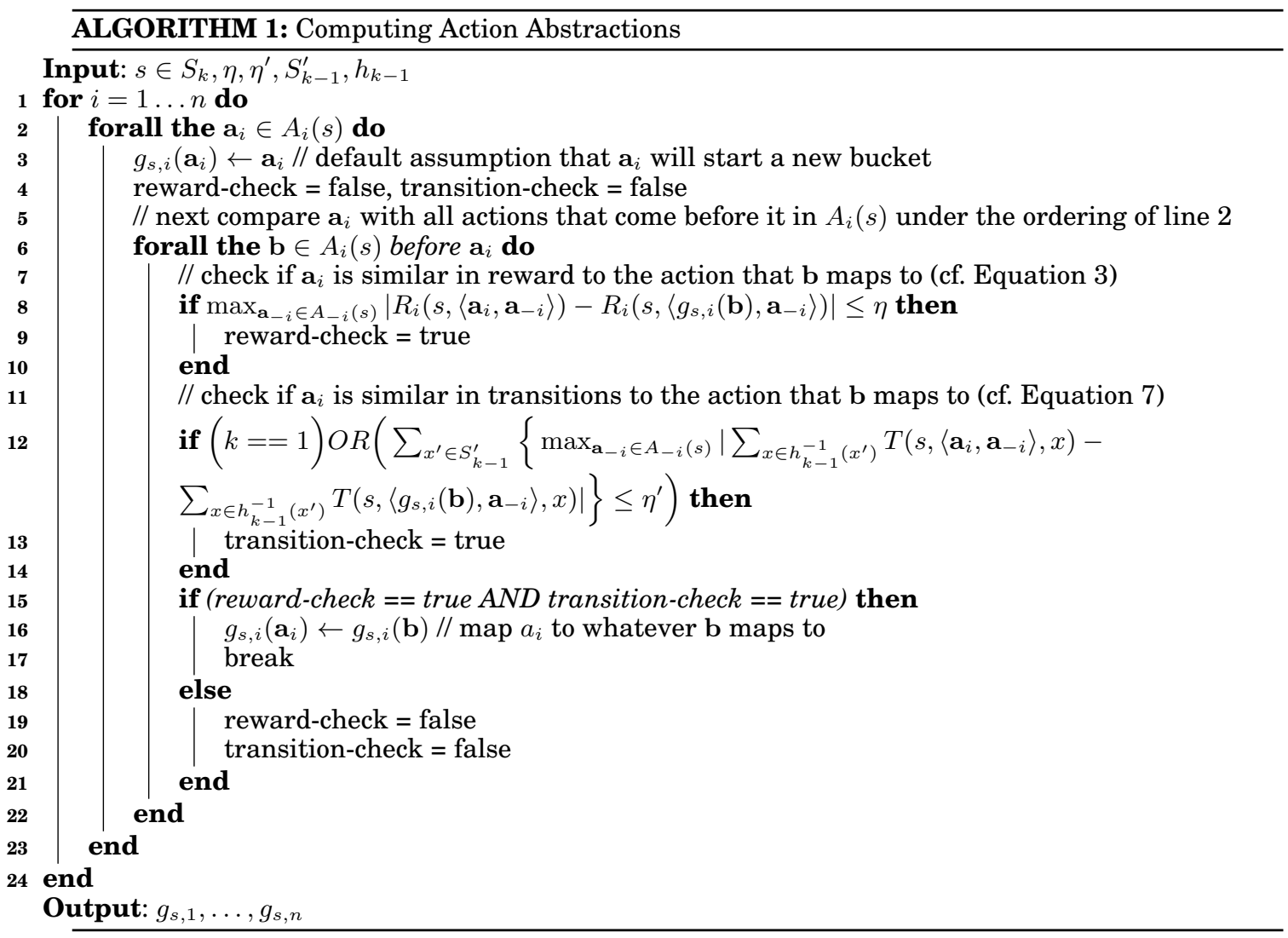

algorithm and if such a matching exists $s$ is mapped (line 14) to the prototype state $h_{k}(x)$. Consequently, Algorithm 2 buckets similar states with the $h$ function mapping each state to a prototype-state for its bucket and is called once for each level (see line 7 of Algorithm 3).

Constructing $M^{\prime}$. The bottom-up single-pass Algorithm 3 first calls the action abstraction algorithm (lines 3-5) with approximation parameters $\frac{\epsilon_{k}^{R}}{2}$ and $\frac{\epsilon_{k}^{T}}{2}$, where the fractions are used to allow for approximation on both sides of the choice of the prototypes in each bucket. Then it calls the state abstraction algorithm (line 7) again with fractional approximation parameters for the same reason as for the action abstraction algorithm. It returns the action-abstraction function $g$ as well as the state-abstraction function $h$. From these the transition probabilities and the reward functions of $M^{\prime}$ can be defined by using the transition probabilities and the reward functions in $M$ for the prototype states and actions defined in the buckets in $h$ and $g$.

6.1.1. Comments on the greedy abstraction algorithm. Algorithm 3 is correct by construction; it yields an $M^{\prime}$ that satisfies the transition and reward approximation bounds specified as inputs to the algorithm. Recall that these reward and transition bounds were chosen to guarantee the upper-bound on the approximation quality of the SPNE found in $M^{\prime}$ when implemented in $M$.

If $\epsilon_{k}^{R}$ and $\epsilon_{k}^{T}$ is set equal to zero for all levels $k$, any abstractions found by Algorithm 3 would be provably lossless. 

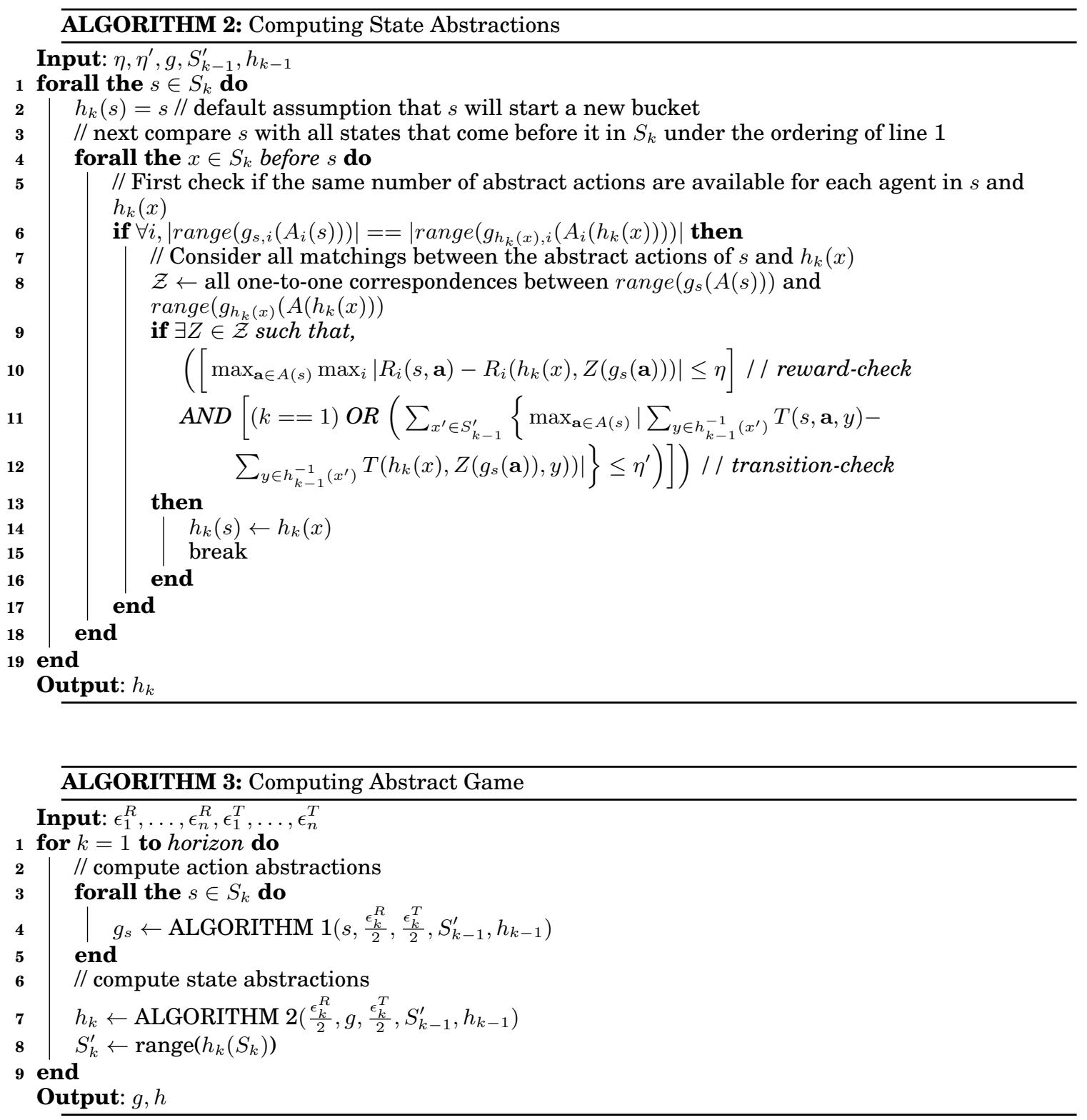

If only action abstraction were desired, this can be obtained easily by simply not calling the state abstraction algorithm (i.e., by deleting line 7 and setting the $h$ function to the identity function). If, on the other hand, only state abstraction were desired, this can be obtained just as easily by not calling the action abstraction algorithm (i.e., by deleting lines 3 to 5 and setting the $g$ function to the identity function).

Finally, we expect the actual regret in $M$ from using the lifted strategies corresponding to the SPNE strategies found in $M^{\prime}$ would typically be far smaller than that implied by the theoretical worst-case upper bounds. We plan to evaluate this empirically in future work. 


\subsection{Bottom-up single-pass level-by-level integer linear program (ILP) for abstraction}

One can ask whether it is possible to write a mathematical program to optimize over entire abstractions. Unfortunately, the constraints in the program would not be linear. (Problems where the constraints and objective are linear tend to be dramatically faster to solve.) For example, the error at level 2 would involve a product of reward error in level 1 and transition probability error in level 2 . In general, with a $k$-level LDAG, the constraints in the mathematical program would be $k$ th-order polynomials.

Therefore, in this section we develop an integer linear program (ILP) that tries to optimize the action and state abstraction within a level, given the abstraction at levels below, and given an error bound allowed for this level. Note that this approach might not yield the best (coarsest in some sense) abstraction for the allowed overall error across levels because, for instance, we do not optimize all levels simultaneously and we assume that the allowable error for each level is given. Nevertheless, as we will see, this approach avoids the need to make many of the decision choices used in the greedy algorithm of the previous section. The ILP is used the same way as the greedy algorithm: bottom level first, then the level above it, and so on.

This ILP chooses prototypical actions (one for each action bucket for each state and agent in the abstraction) and prototypical states (one for each state bucket in the abstraction)_rather than generating new actions or new states to be the prototypes.

By construction, the ILP will generate an abstraction that honors the bounds.

We will present the ILP for optimizing an interior level $k$. The bottom level is simpler because there are no transitions left; we will not present the ILP for that.

The following constraint allows the ILP to split the overall allowed error for this level, $\epsilon_{k}$ (which is given as an input to the ILP) between reward error and transition error. (The greedy algorithm of the previous section requires both of these numbers as input. In other words, it does not handle this splitting.)

$$
\varepsilon_{k}^{R}+\varepsilon_{k}^{T} \bar{W} \leq \epsilon_{k}
$$

Here, $\overline{W_{k}}=\max _{\sigma^{\prime}} \bar{W}_{k}^{\sigma^{\prime}}$. (Note that this is a conservative multiplier; by using a less conservative-i.e., less aggregated-multiplier, it might be possible to get coarser abstractions while still satisfying the overall error bound.)

The following constraints allow the ILP to split the error between action abstraction and state abstraction. (In contrast, the greedy algorithm of the previous section hardwires these splits 50-50.)

$$
\varepsilon_{k}^{\text {state }, R}+\varepsilon_{k}^{\text {action }, R} \leq \varepsilon_{k}^{R} \text { and } \varepsilon_{k}^{\text {state }, T}+\varepsilon_{k}^{\text {action }, T} \leq \varepsilon_{k}^{T}
$$

Now, we define the following helpful auxiliary variables:

$$
\begin{aligned}
\alpha_{s}= & 1 \text { if state } s \text { is selected as a state prototype, and } 0 \text { otherwise. } \\
\beta_{s, a, i}= & 1 \text { if action } a \text { is selected as an action prototype for agent } i \text { in state } s, \text { and } \\
& 0 \text { otherwise. }
\end{aligned}
$$

The objective in the ILP can be any linear function of the variables. Our goal is to generate a coarse abstraction, so the equilibrium finding in the abstracted game would be scalable. However, what kind of abstraction is good for scalability may depend on the problem (e.g., whether the game is zero-sum or not) and on the equilibriumfinding algorithm. One family of proxies for this is the size of the resulting abstraction as measured by some linear combination of the number of states and actions in the abstracted game:

$$
\min \sum_{s} \alpha_{s}+\rho \sum_{s, a, i} \beta_{s, a, i}, \text { for any constant } \rho
$$


(Note that we could also have different $\rho$ 's for different agents at different states. Also, we could use a different $\rho$ when running the ILP at different levels of the LDAG.)

Next, we tie the elements to the prototypes using additional auxiliary variables:

$H_{s, t}=1$ if state $s$ maps to prototype $t$, and 0 otherwise.

$G_{a, b, s, i}=1$ if action $a$ maps to action prototype $b$ in state $s$ for agent $i$, and 0 otherwise.

We do the tying in the ILP using the following constraints:

$$
\begin{aligned}
\forall s, \sum_{t} H_{s, t}=1 \quad / / \text { Each state maps to exactly one prototype state } \\
\quad \text { (possibly itself). } \\
\forall s, i, a \in A_{i}(s), \sum_{b} G_{a, b, s, i}=1 \quad / / \text { Each action maps to exactly one prototype action. }
\end{aligned}
$$

Next, we tie the above mappings to the $\alpha$ 's and $\beta$ 's:

$$
\forall t, \sum_{s} H_{s, t} \leq \alpha_{t} / / \text { Only selected state prototypes can be mapped to. }
$$

$\forall s, i, b \in A_{i}(s), \sum_{a} G_{a, b, s, i} \leq \beta_{s, b, i} / /$ Only selected action prototypes can be mapped to.

Then, we have to make sure that in the abstracted game, all actions within an information set have the same actions. In other words, states can be bundled only if they have the same abstracted action set:

$$
\forall s, t,\left(H_{s, t}=1 \quad \Rightarrow \quad \forall i, a \in A_{i}(s), b \in A_{i}(s), G_{a, b, s, i}=G_{a, b, t, i}\right) .
$$

This can be written in the ILP, for example, using the following two sets of constraints:

$$
\begin{aligned}
& \forall s, t, i, a \in A_{i}(s), b \in A_{i}(s), G_{a, b, s, i} \leq G_{a, b, t, i}+\left(1-H_{s, t}\right) \\
& \forall s, t, i, a \in A_{i}(s), b \in A_{i}(s), G_{a, b, t, i} \leq G_{a, b, s, i}+\left(1-H_{s, t}\right) .
\end{aligned}
$$

Like in the greedy algorithm in the previous section, the action abstraction reward checks are

$$
\begin{aligned}
& \forall s, i, a \in A_{i}(s), b \in A_{i}(s), \\
& G_{a, b, s, i}=1 \quad \Rightarrow \quad \max _{\mathbf{a}_{-i} \in A_{-i}(s)}\left|R_{i}\left(s,\left\langle a, \mathbf{a}_{-i}\right\rangle\right)-R_{i}\left(s,\left\langle b, \mathbf{a}_{-i}\right\rangle\right)\right| \leq \varepsilon_{k}^{a c t i o n}, R .
\end{aligned}
$$

(Note that the only variable on the right hand side of the implication is $\varepsilon_{k}^{a c t i o n, R}$.) These conditions can be written as ILP constraints, for example, as follows:

$$
\begin{aligned}
& \forall s, i, a \in A_{i}(s), b \in A_{i}(s), \\
& \quad \max _{\mathbf{a}_{-i} \in A_{-i}(s)}\left|R_{i}\left(s,\left\langle a, \mathbf{a}_{-i}\right\rangle\right)-R_{i}\left(s,\left\langle b, \mathbf{a}_{-i}\right\rangle\right)\right| \leq \varepsilon_{k}^{a c t i o n, R}+M\left(1-G_{a, b, s, i}\right),
\end{aligned}
$$

where $M$ is a large number (as is standard in integer programming).

Similarly, the action abstraction transition error checks can be written as ILP constraints as follows:

$$
\begin{aligned}
& \forall s, i, a \in A_{i}(s), b \in A_{i}(s), \\
& \sum_{x^{\prime} \in S_{k-1}^{\prime}}\left\{\max _{\mathbf{a}_{-i} \in A_{-i}(s)}\left|\sum_{s^{\prime} \in h_{k-1}^{-1}\left(x^{\prime}\right)} T\left(s,\left\langle a, \mathbf{a}_{-i}\right\rangle, s^{\prime}\right)-\sum_{s^{\prime} \in h_{k-1}^{-1}\left(x^{\prime}\right)} T\left(s,\left\langle b, \mathbf{a}_{-i}\right\rangle, s^{\prime}\right)\right|\right\} \\
& \leq \varepsilon_{k}^{a c t i o n, T}+M\left(1-G_{a, b, s, i}\right) .
\end{aligned}
$$


In principle, the state abstraction reward error checks are as follows, for each state $s$ and the prototype state $t$ of the state bucket that $s$ belongs to:

$$
\max _{\mathbf{a} \in A(s)} \max _{i}\left|R_{i}(s, \mathbf{a})-R_{i}\left(t, g_{s}(\mathbf{a})\right)\right| \leq \varepsilon_{k}^{\text {state }, R} .
$$

However, we have to be careful because the action abstraction function $g$ is not a constant now, but endogenous to the ILP. (The helpful side of this is that-unlike in the greedy state abstraction algorithm in the previous section (ALGORITHM 2)-we do not have to have a $Z$-function that studies the one-to-one correspondences between action in $s$ and $t$ for agent $i$ : the ILP will automatically generate a $g$ where the actions match.) We can write these checks as ILP constraints as follows. The idea is that we write the constraints down for all possible abstractions, and then relax them (using the big- $M$ 's) for the abstractions that the ILP does not choose:

$$
\begin{aligned}
& \forall s, t, i, \mathbf{a} \in \mathbf{A}(s), \mathbf{b} \in \mathbf{A}(s), \\
& \left|R_{i}(s, \mathbf{a})-R_{i}(t, \mathbf{b})\right| \leq \varepsilon_{k}^{\text {state }, R}+M\left(1-H_{s, t}\right)+\sum_{j} M\left(1-G_{\mathbf{a}_{j}, \mathbf{b}_{j}, s, j}\right) .
\end{aligned}
$$

Finally, the state abstraction transition error checks are conceptually as follows. They check whether the transitions are similar for $s$ and the prototype state $t$ of the state bucket that $s$ belongs to:

$$
\sum_{x^{\prime} \in S_{k-1}^{\prime}}\left\{\max _{\mathbf{a} \in A(s)}\left|\sum_{s^{\prime} \in h_{k-1}^{-1}\left(x^{\prime}\right)} T\left(s, \mathbf{a}, s^{\prime}\right)-\sum_{s^{\prime} \in h_{k-1}^{-1}\left(x^{\prime}\right)} T\left(t, g_{s}(\mathbf{a}), s^{\prime}\right)\right|\right\} \leq \varepsilon_{k}^{\text {state }, T} .
$$

Now, again, $g$ is endogenous to the ILP. We can write the above conditions as ILP constraints as follows:

$$
\begin{aligned}
& \forall s, t, \mathbf{a} \in \mathbf{A}(s), \mathbf{b} \in \mathbf{A}(s), \\
& \sum_{x^{\prime} \in S_{k-1}^{\prime}}\left\{\max _{\mathbf{a} \in A(s)}\left|\sum_{s^{\prime} \in h_{k-1}^{-1}\left(x^{\prime}\right)} T\left(s, \mathbf{a}, s^{\prime}\right)-\sum_{s^{\prime} \in h_{k-1}^{-1}\left(x^{\prime}\right)} T\left(t, \mathbf{b}, s^{\prime}\right)\right|\right\} \\
& \leq \varepsilon_{k}^{\text {state }, T}+M\left(1-H_{s, t}\right)+\sum_{j} M\left(1-G_{\mathbf{a}_{j}, \mathbf{b}_{j}, s, j}\right) .
\end{aligned}
$$

This completes the construction of the ILP. Note that the ILP handles action and state abstraction simultaneously, unlike the greedy algorithm of the previous section that does one of these first without looking at the other, and then does the other.

\subsection{Discussion of the abstraction algorithms and problem complexity}

Both the ILP and the greedy algorithm satisfy the accuracy bounds by construction. For any constant number of agents, the ILP is of polynomial size. However, the withinlevel abstraction problem that both algorithms are trying to solve is hard in the worst case:

PROPOSITION 6.1. The (decision version of the) within-level abstraction optimization problem that the ILP and the greedy algorithm are trying to solve (where the state and action prototypes have to be selected from among the actual states and actions, respectively) is NP-complete. This is the case even if we only want to do action abstraction (i.e., no state abstraction), even if there are only two agents and one state in the game, and the game is only played for one step.

PROOF. It is easy to check the solution, so the problem is in NP. What remains to be shown is that it is NP-hard. We reduce from SET COVER, which is NP-complete. 
There we are given a set $\mathcal{S}=\left(S_{1}, \ldots, S_{r}\right)$ and a ground set of elements $E=\{1, \ldots, q\}$. Each set $S_{i}$ contains some subset of $E$. The question is whether all the items in $E$ can be covered using only $k$ sets from $\mathcal{S}$.

Now, we can solve this problem by considering the abstraction problem of a 2 -agent game where there is only one state and one step. The two agents are an attacker and a defender. The attacker's action set is a set of locations $E=\{1, \ldots, q\}$. The defender's action set is $\mathcal{S}=\left(S_{1}, \ldots, S_{r}\right)$, where each $S_{i} \subseteq E$ and $\bigcup_{i} S_{i}=E$. The payoffs in this game are such that the attacker gets $M$ if he attacks a location that the defender does not defend, and 0 otherwise (here, $M$ is very large). The defender gets 1 if he defends successfully and 0 otherwise.

We set the target accuracy in the abstraction algorithm so that the defender cannot be guaranteed to lose, that is, each location is coverable by some action of the defender. (It is possible to set the target accuracy this way because the attacker's payoffs dominate the defender's.) We ask the abstraction algorithm whether such an abstraction exists with $k$ actions for the defender. This is exactly SET COVER.

In general, the ILP might not return the coarsest abstraction for the overall allowed target accuracy for at least the following reasons: 1 ) it optimizes one level at a time, 2) it assumes that the overall target accuracy is somehow exogenously divided into target accuracies for all levels, 3) the analysis in the theory part of this paper is not tight (e.g., there is slack in the inequalities regarding transition probability error such as in line 5 of Equation 13), and 4) in the ILP, the multiplier $\bar{W}$ that is used to multiply the transition probability error is conservative (and this might cause the greedy algorithm to occasionally produce better abstractions than the ILP). However, the game used in the proof of Proposition 6.1 has only one level, one state, and no transitions, so none of these limitations apply to it, and the ILP will find the optimal abstraction for games in that class. The NP-hardness reduction in the proof is from SET COVER, which cannot be approximated in worst-case polynomial time to an approximation ratio better than a constant times $\ln q$ unless $\mathrm{P}=\mathrm{NP}$ [Alon et al. 2006]. Therefore, the gap in solution quality-in terms of the size of the (action) abstraction for the level-found by the greedy algorithm (which runs in polynomial time) versus the ILP (which finds an optimal solution in this game class) cannot be bounded by any constant. So, no matter how many orders of magnitude difference one wants to demonstrate, an input that demonstrates at least that big a difference exists. Therefore the difference in solution quality between the two algorithms depends completely on the game that one is interested in.

In general, the abstraction problem being hard in the worst case does not mean that the ILP will be slow in practice. In fact, it has been shown experimentally that withinlevel abstraction optimization ILPs run fast (in less than a second) even in the large (in a different type of game state abstraction problem) [Gilpin and Sandholm 2007a]. How fast the ILP will run in practice will depend on the game.

Running a polynomial-time abstraction algorithm (such as our greedy algorithm), or an abstraction algorithm that runs fast in practice though not being polynomial in the worst case, can be very helpful as a preprocessor. These algorithms run in polynomial time in the entire stochastic game, while the (Nash) equilibrium-finding algorithm can take exponential time just to solve one stage game-because solving a normal form game is PPAD-complete (even with two agents and all payoffs in $\{0,1\}$ ) [Daskalakis et al. 2009; Chen et al. 2009; Abbott et al. 2005].

\section{CONCLUSIONS AND FUTURE RESEARCH}

Abstraction followed by equilibrium finding has emerged as a leading approach to solving games. Lossless abstraction typically yields games that are still too large to solve, 
so lossy abstraction is needed. Unfortunately, prior lossy game abstraction algorithms have no guarantees on solution quality.

We developed a framework that enables the design of lossy game abstraction algorithms with guarantees on solution quality. It simultaneously handles state and action abstraction. We defined a measure of reward approximation error and transition probability error achieved by state and action abstraction in stochastic games such that the regret of the equilibrium found in the abstract game when implemented in the original, unabstracted game is upper-bounded by a function of those measures.

We then developed the first lossy game abstraction algorithms with bounds on solution quality. Both of them work level-by-level up from the end of the game. One of the algorithms is greedy and the other is an integer linear program. We also proved that the abstraction problem is NP-complete (even with just action abstraction, 2 agents, and a 1-step game), but want to point out that this does not mean that the game abstraction problems that occur in practice cannot be solved quickly.

The obvious stream of applications of this work is to develop better (faster and/or higher-accuracy) game-solving algorithms by having better automated abstraction algorithms. However, the work has an additional important potential application stream in modeling. All models are abstractions of reality. As one models a real-world situation formally as a game, one faces modeling choices. What aspects of the real world situation should be captured in the model? How detailed should one make the signal space? For example, how should one discretize state if the game solver requires discrete states? How should one discretize the action space when there are too many (and possibly infinitely many) actions in reality but one wants to use a scalable game solver that assumes a finite action space? Such modeling questions are really questions about abstraction, and the work in this paper will help pave the way to answering those questions in a rigorous way, whether the modeling is done by human or by machine.

There is ample scope for future research. Both of our algorithms obtain action and state abstraction by removing some actions and states and keeping others. Our theoretical results do not assume this form of abstraction and other more general forms of abstraction (with automated state and action generation) may yield smaller abstract games. We also believe that our results will extend to sequential games, at least as long as the information sets form a layered directed acyclic graph.

\section{REFERENCES}

AввотT, T., KANE, D., AND VALiant, P. 2005. On the complexity of two-player winlose games. Annual Symposium on Foundations of Computer Science (FOCS).

Alon, N., Moshrovitz, D., AND SAFRA, S. 2006. Algorithmic construction of sets for k-restrictions. ACM Trans. Algorithms 2, 2, 153-177.

ARCHIBALD, C. AND ShohAM, Y. 2009. Modeling billiards games. International Conference on Autonomous Agents and Multi-Agent Systems (AAMAS).

Billings, D., Burch, N., Davidson, A., Holte, R., Schaeffer, J., SchauenBERG, T., AND SzAFRON, D. 2003. Approximating game-theoretic optimal strategies for full-scale poker. International Joint Conference on Artificial Intelligence (IJCAI).

Chen, X., Deng, X., And Teng, S.-H. 2009. Settling the complexity of computing two-player Nash equilibria. Journal of the ACM 56, 3.

Daskalakis, C., GoldberG, P., AND PAPADimitriou, C. 2009. The complexity of computing a Nash equilibrium. SIAM Journal on Computing 39, 1, 195-259.

GANZFried, S., SANDHOlm, T., AND WAUGH, K. 2012. Strategy purification and thresholding: Effective non-equilibrium approaches for playing large games. International Conference on Autonomous Agents and Multi-Agent Systems (AAMAS).

GILPIN, A. AND SANDHOLM, T. 2006a. A competitive Texas Hold'em poker player via 
automated abstraction and real-time equilibrium computation. National Conference on Artificial Intelligence (AAAI).

GILPIN, A. AND SANDHOLM, T. 2007a. Better automated abstraction techniques for imperfect information games, with application to Texas Hold'em poker. International Conference on Autonomous Agents and Multi-Agent Systems (AAMAS).

GILPIN, A. AND SANDHOLM, T. 2007b. Lossless abstraction of imperfect information games. Journal of the ACM 54, 5.

GILPIN, A. AND SANDHOLM, T. 2008. Expectation-based versus potential-aware automated abstraction in imperfect information games: An experimental comparison using poker. National Conference on Artificial Intelligence (AAAI). Short paper.

Gilpin, A., SAndholm, T., AND Sørensen, T. B. 2007. Potential-aware automated abstraction of sequential games, and holistic equilibrium analysis of Texas Hold'em poker. National Conference on Artificial Intelligence (AAAI).

Gilpin, A., Sandholm, T., AND Sørensen, T. B. 2008. A heads-up no-limit Texas Hold'em poker player: Discretized betting models and automatically generated equilibrium-finding programs. International Conference on Autonomous Agents and Multi-Agent Systems (AAMAS).

Givan, R., DEAN, T., AND GREIG, M. 2003. Equivalence notions and model minimization in markov decision processes. Artificial Intelligence 147, 1-2, 163-223.

HaWkin, J., Holte, R., AND SzAFron, D. 2011. Automated action abstraction of imperfect information extensive-form games. National Conference on Artificial Intelligence (AAAI).

JiAng, A. X. AND Leyton-Brown, K. 2011. Polynomial-time computation of exact correlated equilibrium in compact games. ACM Conference on Electronic Commerce.

Kearns, M., Mansour, Y., AND NG, A. Y. 1999. A Sparse Sampling Algorithm for Near-Optimal Planning in Large Markov Decision Processes. International Joint Conference on Artificial Intelligence.

Lipton, R., MARKAKIS, E., AND Mehta, A. 2003. Playing large games using simple strategies. ACM Conference on Electronic Commerce (EC).

Littman, M. AND Stone, P. 2003. A polynomial-time Nash equilibrium algorithm for repeated games. ACM Conference on Electronic Commerce (EC).

RAVINDRAN, B. 2004. An algebraic approach to abstraction in reinforcement learning. Ph.D. thesis, University of Massachusetts, Amherst, MA.

Schnizlein, D., Bowling, M., AND Szafron, D. 2009. Probabilistic state translation in extensive games with large action sets. International Joint Conference on Artificial Intelligence (IJCAI).

SHI, J. AND LitTMAN, M. 2002. Abstraction methods for game theoretic poker. Revised Papers from International Conference on Computers and Games. Springer.

SoRG, J. AND SingH, S. 2009. Transfer via soft homomorphisms. International Conference on Autonomous Agents and Multiagent Systems (AAMAS).

WaUgh, K., Schnizlein, D., Bowling, M., AND Szafron, D. 2009a. Abstraction pathologies in extensive games. International Conference on Autonomous Agents and Multi-Agent Systems (AAMAS).

WaUgh, K., Zinkevich, M., Johanson, M., Kan, M., Schnizlein, D., AND BowlING, M. 2009b. A practical use of imperfect recall. Symposium on Abstraction, Reformulation and Approximation (SARA).

Wellman, M. 2006. Methods for empirical game-theoretic analysis (extended abstract). National Conference on Artificial Intelligence (AAAI).

Zinkevich, M., Bowling, M., Johanson, M., AND PiCCIOne, C. 2007. Regret minimization in games with incomplete information. Annual Conference on Neural Information Processing Systems (NIPS). 